\title{
Association between Cholesterol-Phospholipid Vesicles and Cholesterol Crystals in Human Gallbladder Bile
}

\author{
Carolin ERIKa Schriever AND DIETER JÜngST \\ Department of Internal Medicine 11, Klinikum Grosshadern, Liniversity of Munich, 8000 Munich 70, Federal Republic of \\ Germany
}

Rapid aggregation of cholesterol-phospholipid vesicles in gallbladder bile seems to be the first event in the production of cholesterol crystals, a prerequisite for cholesterol gallstone formation. We examined the amount of these vesicles in $\mathbf{3 3}$ human gallbladder biles in relation to biliary lipid composition and to the presence of cholesterol crystals. Biliary microscopy detected cholesterol crystals in all 19 biles from patients with cholesterol gallstones but in none of 14 biles from patients with pigment stones. Gel chromatography was used to separate vesicles and micelles in the native bile with an eluting buffer containing $10 \mathrm{~m} M$ sodium cholate to prevent disruption of micellar lipids. Cholesterol, phospholipid and bile salt concentrations were measured in every fraction collected. Bile acid, phospholipid, cholesterol and total lipid concentrations were not significantly different in samples with and without cholesterol crystals. The cholesterol saturation index $(1.4 \pm 0.11$ vs. $1.0 \pm 0.08)$ was significantly $(p<0.01)$ higher in biles with crystals than without crystals. Gel filtration revealed a vesicular peak in addition to micellar fraction in $18(23.1 \pm 3.2 \%$ of total cholesterol) of the 19 biles with crystals but only in three $(15.7 \pm 2.4 \%$ of total cholesterol) of 14 biles without crystals. There was no relation between biliary lipid concentration or the cholesterol saturation index and the percentage of vesicular cholesterol in biles with or without crystals. The close association of vesicles and crystals in human gallbladder bile supports the contention that vesicles are important in the initial nucleation of cholesterol monohydrate crystals.

Cholesterol-phospholipid vesicles and mixed bile saltphospholipid-cholesterol micelles are the major transport forms of cholesterol in human bile. Vesicles were first demonstrated by quasielastic light scattering in model bile solutions (1) and in fresh human hepatic bile (2).

Received February 24, 1988; accepted October 6, 1988.

This work was presented in part at the Annual Meeting of the American Association for the Study of Liver Diseases, Chicago, Illinois, October, 1987, and has previously been published in abstract form (Hepatology 1987; 7:1111).

The results of this study are part of the thesis of C. E. S. with the title: "Untersuchung von vesikulären und mizellären Lipidtransportformen in der menschlichen Galle. Dissertation der Medizinischen Fakultät der Ludwig-Maximilians-Universität, in Vorbereitung."

Address reprint requests to: Dieter Jüngst, M.D., Department of Medicine II, Klinikum Grosshadern, Marchioninistr. 15, 8 Munich 70, Federal Republic of Germany.
Previous studies indicated an important role for vesicles in the formation of cholesterol crystals $(3,4)$. Vesicle aggregation is followed by cholesterol monohydrate nucleation $(3,5,6)$. Gallbladder bile from patients with cholesterol gallstones almost always contains cholesterol crystals (7-10), and bile from these patients nucleates cholesterol much faster than bile from patients with pigment stones or from healthy controls $(11,12)$. Following separation by gel filtration, vesicular fractions show rapid nucleation of cholesterol crystals, but nucleation is much slower in mixed micellar fractions (4). Very recently, the same authors (13) demonstrated that the rate of cholesterol monohydrate crystal formation seemed to be directly related to the amount of cholesterol transported in vesicles.

These findings point to a crucial role for cholesterolphospholipid vesicles in the crystallization of biliary cholesterol and the subsequent formation of cholesterol gallstones. To evaluate this hypothesis, we determined the amount of cholesterol present in vesicles from bile samples with and without cholesterol crystals.

\section{MATERIALS AND METHODS}

Thirty-three patients with cholecystolithiasis undergoing elective cholecystectomy were studied. During surgery, bile samples were aspirated under sterile conditions by puncture of the gallbladder after ligation of the cystic duct. Particular care was taken to aspirate gallbladder bile completely, in order to avoid effects of stratification of bile (14).

Polarizing Light Microscopy. Bile samples were stored in the dark at $37^{\circ} \mathrm{C}$. Immediately after bile collection, samples were mixed thoroughly and then analyzed using a polarizing light microscope. Cholesterol crystals were identified by their characteristic birefringent parallelogram structure, often with a small notch in one corner. To obtain crystal-free bile, the samples were ultracentrifuged at $100,000 \times g$ in a Beckman ultracentrifuge (Model L5-65, rotor Ti-50, Beckman Instruments, Fullerton, CA). The supernatant and sediment were then reexamined for the presence of cholesterol crystals.

Lipid Analysis. For analysis of biliary lipids, duplicate aliquots were stored at $-20^{\circ} \mathrm{C}$. Cholesterol was measured colorimetrically after extraction with petrol ether (15). Phospholipids were determined using the method of Fiske-Subbarow (16), and total bile salts were analyzed with a slight modification of the $3 \alpha$-hydroxysteroid dehydrogenase method (17). The cholesterol saturation index was calculated by dividing the cholesterol concentration by the maximum cholesterol solubil- 
TABLE 1. Lipid composition, total lipids and cholesterol saturation index in 19 gallbladder biles with and 14 gallbladder biles without cholesterol crystals

\begin{tabular}{lccrr}
\hline \multicolumn{1}{c}{ Gallbladder biles } & $\begin{array}{c}\text { Bile acid } \\
\text { (mmoles/liter) }\end{array}$ & $\begin{array}{c}\text { Phospholipid } \\
\text { (mmoles/liter) }\end{array}$ & $\begin{array}{c}\text { Cholesterol } \\
\text { (mmoles/liter) }\end{array}$ & $\begin{array}{c}\text { Total lipids } \\
\text { (gm/dl) }\end{array}$ \\
\hline With cholesterol crystals & $93.2 \pm 8.1^{a}$ & $28.4 \pm 3.1$ & $11.3 \pm 1.1$ & $7.1 \pm 0.6$ \\
Without cholesterol & $89.2 \pm 10.5$ & $29.3 \pm 4.0$ & $9.2 \pm 1.8$ & $7.0 \pm 0.8$ \\
$\quad$ srystals & & & & \\
\hline
\end{tabular}

${ }^{a}$ Mean \pm S.E.

${ }^{b}$ Significant difference $(p<0.01)$.

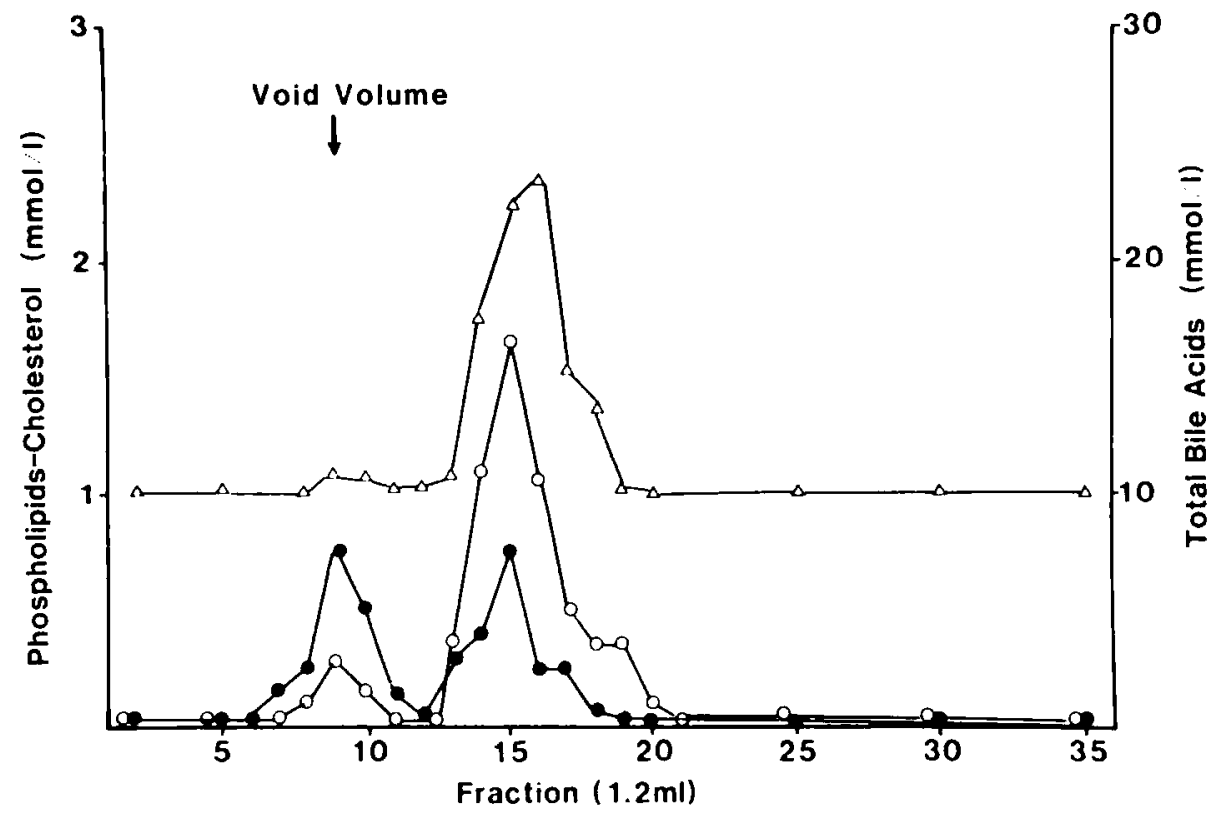

Fig. 1. Gel filtration of a gallbladder bile with cholesterol crystals from a patient with cholesterol gallstones. The elution profile shows the measured amounts of total bile acids $(\Delta)$, cholesterol (O) and phospholipids $(O)$ in mmoles per liter.

ity according to Carey and Small (21) and corrected for the total lipid content of each individual bile (18).

Separation of Vesicles and Micelles. Micelles were separated from vesicles by gel filtration as described by Sömjen and Gilat (19). Glass columns, $30 \times 1.2 \mathrm{~cm}$, were filled with Sephacryl S-300 superfine gel containing $0.04 \%$ sodium azide in distilled water (Pharmacia AB, Uppsala, Sweden). The void volume of the column was determined with $2 \%$ Dextran Blue 2000 (Pharmacia). After ultracentrifugation of the bile, $0.5 \mathrm{ml}$ of the isotropic phase was applied to the column and eluted in 1.2-ml fractions using Tris- $\mathrm{HCl}$ buffer $(\mathrm{pH} 8.0$ ) containing 10 $\mathrm{m} M$ sodium cholate to prevent disruption of micellar lipids. Thirty-five fractions were collected and analyzed for cholesterol, phospholipid and bile salt content. Sodium cholate, an unconjugated bile salt, was used in the chromatography elution buffer according to the original method of Sömjen and Gilat (19). Control experiments with a conjugated bile acid, sodium taurocholate, in the elution buffer revealed nearly identical results for the separation of the vesicular and micellar fractions of human gallbladder bile.

Stone Analysis. Gallbladder stones were removed during surgery, washed with distilled water, dried and weighed. The cholesterol content was measured chemically and expressed as percentage of the dry weight.

Statistical Analysis. The data are given as means \pm S.E. The statistical significance of differences between means was assessed with the t test.

\section{RESULTS}

The gallbladder biles obtained from 33 patients during cholecystectomy were separated into two groups accord- ing to the presence or absence of microscopic cholesterol crystals. All of the 19 patients with cholesterol gallstones displayed multiple cholesterol crystals of typical shape in their native biles and in the sediment after ultracentrifugation. The cholesterol content of the stones varied from 58 to $77 \%$ (mean: $71 \pm 1.4 \%$ cholesterol). In the other group of 14 patients, no cholesterol crystals could be detected in the native biles nor in the sediment after ultracentrifugation. These biles were obtained from patients with pigment stones with a cholesterol content between 0 and $6 \%$ (mean: $3 \pm 1.0 \%$ cholesterol).

The mean concentrations $\pm \mathrm{S}$.E. of bile acids, phospholipids, cholesterol, total lipids as well as the cholesterol saturation index (CSI) in gallbladder biles with and without cholesterol crystals are illustrated in Table 1. No significant difference for biliary lipids was obtained between biles with and without cholesterol crystals. However, the CSI was significantly higher $(p<0.01)$ in gallbladder biles with crystals than in biles without crystals.

Figure 1 displays the elution profile of a gallbladder bile from a patient with cholesterol gallstones and crystals. In this example, the vesicular peak contained $47 \%$ of the total biliary cholesterol. In 18 of 19 gallbladder biles with cholesterol crystals, a vesicular peak could be separated from a micellar peak. By contrast, the gallbladder biles from patients with pigment stones and without cholesterol crystals usually showed a different elution profile after gel filtration. Although a vesicular 
FIG. 2. Gel filtration of a gallbladder bile without cholesterol crystals from a patient with pigment stones. The elution profile shows the measured amounts of total bile acids $(\Delta)$, cholesterol $(\bullet)$ and phospholipids $(O)$ in mmoles per liter.

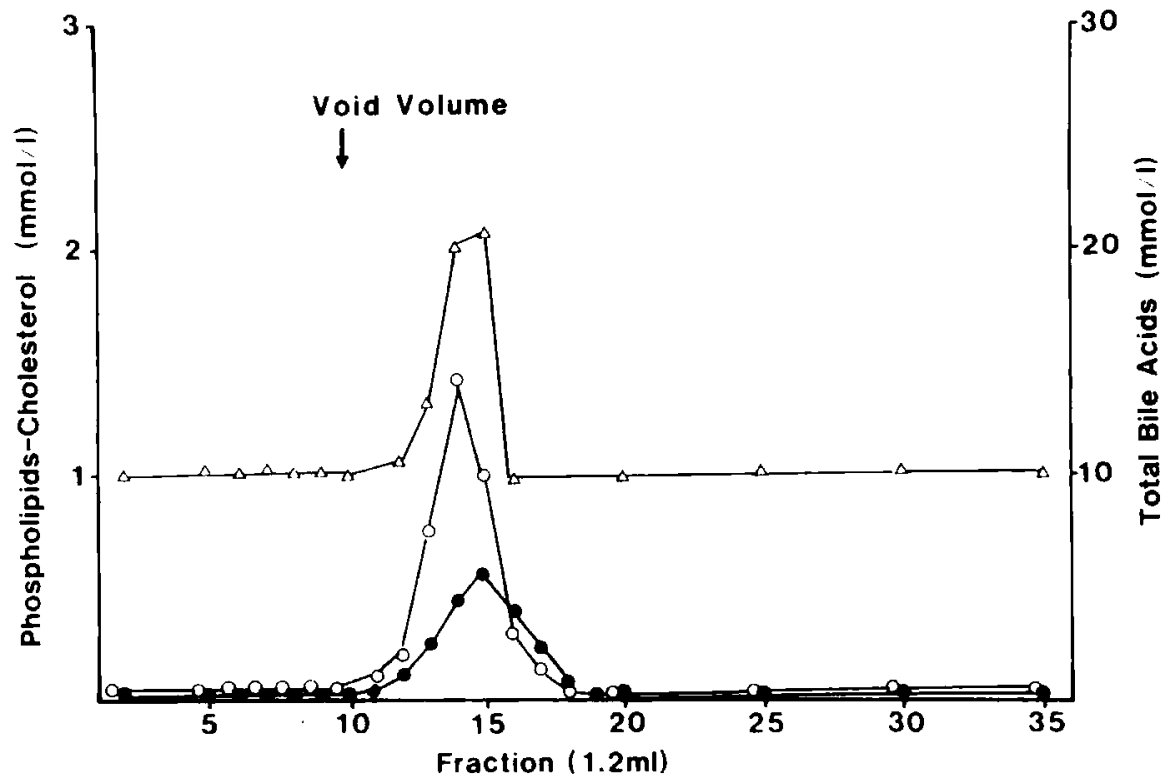

TABLE 2. Cholesterol/phospholipid ratio in vesicles and micelles and percentage of total cholesterol in vesicles in 19 gallbladder biles with and 14 gallbladder biles without Figure 2. The recovery of cholesterol after gel filtration was above $90 \%$.

To evaluate the lipid content in each peak, the measurements in all fractions pertaining to the peak were added and related to the native lipid concentrations. Table 2 shows the vesicle and micelle composition and cholesterol distribution in the four different groups of biles.

The relationship between the cholesterol saturation index and the percentage of vesicular cholesterol in gallbladder biles with and without cholesterol crystals is illustrated in Figure 3a. Although there was a considerable overlap of the CSIs in samples with and without crystals, marked supersaturation above 1.6 was seen only in biles with cholesterol crystals and marked unsaturated biles below 0.8 occurred only in the group without crystals. There were three supersaturated biles without crystals or vesicles from patients with pigment stones. The three biles which contained vesicles but no crystals were markedly supersaturated or just saturated with CSIs of approximately 1.0 . Five biles with crystals from patients with cholesterol gallstones contained measurable amounts of vesicular cholesterol despite slight undersaturation (0.8 to 0.9$)$. The amount of cholesterol carried in vesicular form ranged from 9 to $56 \%$ of the total biliary cholesterol, and no clear relation to the degree of saturation could be determined.

Figure $3 \mathrm{~b}$ illustrates the relationship between the total lipid concentrations and the percentage of the total biliary cholesterol carried in vesicles.

Total lipid concentrations were similar in samples with and without cholesterol crystals and ranged between 3.5 and $12.2 \mathrm{gm}$ per $\mathrm{dl}$. There were five biles in both groups with low total lipid concentration between 3.5 and $5 \mathrm{gm}$ per dl. In these samples, cholesterol vesicles occurred only in biles with crystals of patients with cholesterol gallstones. No relation between the amount of cholescholesterol crystals

\begin{tabular}{|c|c|c|c|c|}
\hline \multirow[b]{2}{*}{ Gallbladder biles } & \multirow{2}{*}{$\begin{array}{c}\text { No. of } \\
\text { patients }\end{array}$} & \multicolumn{2}{|c|}{ CH/PL ratio ${ }^{a}$} & \multirow{2}{*}{$\begin{array}{c}\text { \% of total } \\
\text { CH in } \\
\text { vesicles }\end{array}$} \\
\hline & & $\begin{array}{c}\text { In } \\
\text { vesicles }\end{array}$ & $\underset{\text { micelles }}{\text { In }}$ & \\
\hline \multicolumn{5}{|l|}{$\begin{array}{l}\text { With cholesterol } \\
\text { crystals }\end{array}$} \\
\hline With vesicles & 18 & $2.0 \pm 0.3^{b}$ & $0.28 \pm 0.03$ & $23.1 \pm 3.2$ \\
\hline Without vesicles & 1. & & 0.38 & \\
\hline \multicolumn{5}{|l|}{$\begin{array}{l}\text { Without cholesterol } \\
\text { crystals }\end{array}$} \\
\hline \multirow[t]{3}{*}{ With vesicles } & 3 & 2.2 & 0.29 & 11.2 \\
\hline & & 3.0 & 0.27 & 17.5 \\
\hline & & 1.1 & 0.28 & 19.0 \\
\hline Without vesicles & 11 & & $0.30 \pm 0.04$ & \\
\hline
\end{tabular}

${ }^{n} \mathrm{CH}=$ cholesterol; $\mathrm{PL}=$ phospholipid.

$f^{h}$ Mean \pm S.E.

terol in vesicles and the total lipid concentrations was apparent.

Figure $3 \mathrm{c}$ relates the total bile concentrations to the percentage of vesicular cholesterol. It is remarkable that some samples with low bile acid content had no vesicles and other biles with high bile salt concentrations (up to 140 mmoles per liter) contained considerable amounts of vesicles.

\section{DISCUSSION}

Previous studies have shown that cholesterol monohydrate crystals are present in the bile of patients with cholesterol gallstones but occur rarely in the bile of patients with pigment stones or no stones (8-10). Until recently, it was generally accepted that the main determinant of crystal formation was the degree to which bile was supersaturated with cholesterol. It has been assumed that cholesterol in bile is entirely solubilized by mixed bile salt-phospholipid micelles and various phase-equi- 

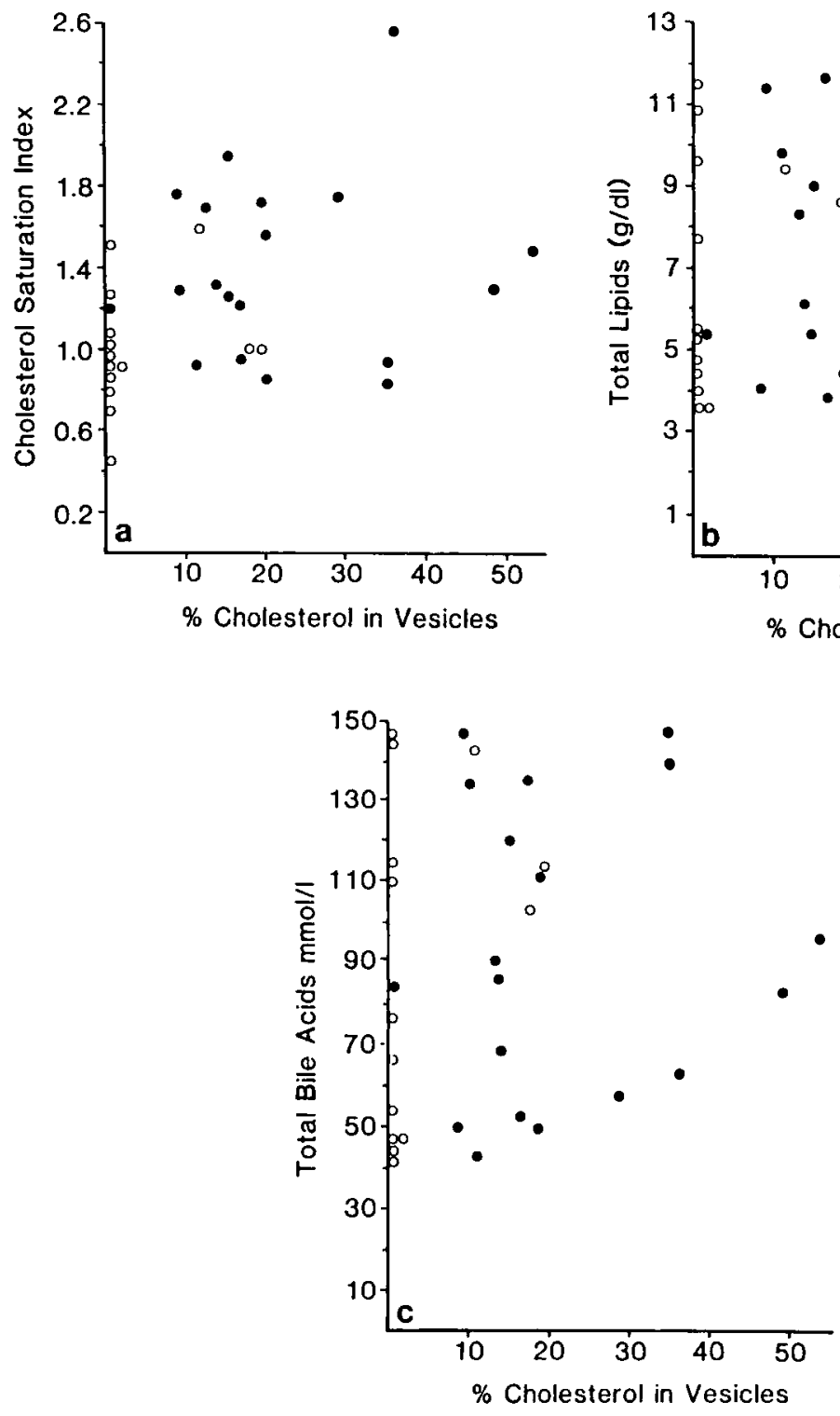

FIG. 3. Relationship between the cholesterol saturation index (a), total lipids (b), total bile acids (c) and the percentage of vesicular cholesterol in human gallbladder biles. The values of 19 gallbladder biles with cholesterol crystals $(\Theta)$ and 14 gallbladder biles without cholesterol crystals $(O)$ are shown. librium diagrams have been devised to describe and predict the solubility in either artificial or native biles in relation to their lipid composition $(20,21)$. A cholesterol saturation index could be calculated, which seems to be correct only if virtually all cholesterol is solubilized and transported by micelles. However, several groups have shown independently that large phospholipid vesicles are present in hepatic as well as in gallbladder bile and provide an additional carrier for cholesterol (2, 19, 2224 ). The calculation of the CSI does not consider that a variable proportion of total cholesterol and phospholipids in bile is carried by nonmicellar particles. Therefore, it is not surprising that results regarding the CSI in gallbladder biles with and without crystals are contradictory $(9,10,20)$. In our study, the concentrations of total bile salts, phospholipids, cholesterol and total lipids were not significantly different in gallbladder biles with and without crystals. However, a significantly higher average CSI was determined in samples with crystals than in those without crystals. Cholesterol monohydrate crystals were present in the gallbladder bile of five of 19 patients with cholesterol gallstones despite a CSI of gallbladder bile which was less than 1.0 (0.8 to 0.9$)$. Spontaneous nucleation of cholesterol crystals is unlikely to occur in unsaturated gallbladder bile. Perhaps the cholesterol crystals detectable at the light microscopic level in the gallbladder bile of patients with cholesterol gallstones are shed from the surface of the stone and do not represent de novo nucleation and crystal growth in these biles. To date, no data are available to differentiate between de novo formation of crystals and crystals which derive from the surface of cholesterol gallstones. However, several studies indicate that the rate of crystal formation and growth is much faster in bile samples from gallstone patients after removal of crystals by ultracentrifugation or ultrafiltration, in comparison to bile samples without cholesterol crystals. The latter were derived from patients with pigment stones or no stones in the gallbladder $(11,25)$. These observations would support the contention that cholesterol crystals seen in biles from patients with cholesterol gallstones are formed de novo and are not derived from the stone surface. Furthermore, Whiting and Watts (25) have shown that gallbladder biles of similar cholesterol saturation, collected from 
patients with and without cholesterol gallstones, have a large difference in their propensity to form cholesterol crystals when incubated in vitro.

These authors determined the cholesterol solubility limit of human gallbladder bile by seeding biles with a small amount of cholesterol crystals. Supersaturated biles supported crystal growth, whereas in undersaturated bile the seed crystals dissolved. In their study of 38 biles, a clear separation was found in the CSI of biles that supported crystal growth and those that did not. However, a surprising finding was that the biliary CSI value corresponding to the solubility limit was not 1.0 but approximately 0.8 when calculated by the method of Carey (18). One possible explanation is that the solubility of cholesterol in human bile is not as great as in the model bile solutions used in determination of the maximum cholesterol solubility and the calculation of the CSI. Our results correspond to these findings, since some bile samples with cholesterol crystals revealed a CSI value between 0.8 and 1.0 .

Other investigators have shown that cholesterol monohydrate crystals appear to nucleate from cholesterol lecithin vesicles in model and native biles $(1,4,6)$.

In gallbladder bile, nucleation of cholesterol was preceded by the formation of vesicles large enough to be visualized by time-lapse, video-enhanced microscopy (3). Subsequently, the vesicles aggregated, and crystals seemed to emerge from these aggregates. Since vesicles seem to play a crucial role in cholesterol nucleation, one would predict that the amount of vesicles would be higher in biles containing crystals than in biles without crystals. Our study conforms to this hypothesis.

Using a standard gel filtration method, cholesterolphospholipid vesicles were detected in 18 of 19 gallbladder biles with crystals but in only 3 of 14 biles without crystals. The amount of cholesterol carried in vesicular form ranged from 9 to $56 \%$ of the total biliary cholesterol in vesicle-containing biles. In a previous study, Sömjen and Gilat (19) examined gallbladder biles from four patients and found between 11 and $52 \%$ of total biliary cholesterol in the vesicular fraction, which was similar to our results. In an extension of these investigations, Harvey et al. (13) determined the amount of cholesterol carried in vesicles in 31 cholesterol gallstone patients and 14 controls without stones. In variance with our findings, cholesterol-phospholipid vesicles were detected in all biles except one of the non-stone group. However, the percentage of cholesterol carried in vesicles in gallbladder bile of stone patients was significantly greater than in controls. The median values were 39 and $25 \%$, respectively. A possible explanation for these discrepant results could be differences in methodology for the determination of cholesterol after gel filtration. In the study of Harvey et al. (13), bile samples were incubated with tritiated cholesterol, $0.1 \mu \mathrm{Ci}$ at $37^{\circ} \mathrm{C}$ for $1.0 \mathrm{hr}$. After the separation of micelles and vesicles by gel filtration, 100- $\mu \mathrm{l}$ aliquots from the $1-\mathrm{ml}$ fractions were counted.

The percentage of cholesterol carried in vesicles was calculated as tritiated dpm in the vesicular peak $\times 100 /$ total tritiated dpm eluted from the column. This kind of calculation seems to be appropriate only if the added tritiated cholesterol reflects exactly the distribution of endogenous cholesterol. In control experiments, we have seen that it is preferable to measure cholesterol in the fractions by chemical means after gel filtration. Nevertheless, the study of Harvey et al. (13) supports the importance of vesicular cholesterol in cholesterol crystal formation in showing that the rate of cholesterol crystal formation is related to the amount of cholesterol transported in vesicles.

However, no information is available to indicate that the process of crystal nucleation which occurs in a crystal-free system (fusion of unilamellar cholesterol-lecithin vesicles, multilamellar vesicle formation and cholesterol monohydrate crystal formation) is the same as that which results in the precipitation of cholesterol onto the growing surface of a preexistent cholesterol crystal. The assumption that the process of nucleation which has been described in gallbladder bile containing gallstones has not been proven, although vesicles seem to be clearly important in the initial nucleation of cholesterol crystals.

The cause of vesicle presence in gallbladder biles containing cholesterol crystals in contrast to biles without crystals is unclear.

Our data show that two patients without cholesterol crystals had supersaturated bile (CSI $=1.51$ and 1.25 ) which did not contain a vesicular fraction (Figure 3a).

Also, five patients with cholesterol crystals had unsaturated biles (CSI $<1.0$ ), yet their gallbladder bile contained a prominent vesicular fraction. The lack of an association between the CSI and the percentage of biliary cholesterol in vesicles in gallbladder bile is an important observation which needs further clarification. Model biles prepared by coprecipitation of the lipids from organic solvents do not contain a vesicular fraction if they are not saturated or supersaturated with cholesterol. The factors which are potentially responsible for the presence of vesicles in unsaturated native biles are not known. There are vesicles in bile up to bile acid concentrations of more than 140 mmoles per liter in single samples (Fig. $3 c)$. In contrast, it is known that vesicles in bile are completely transformed to micelles during gel filtration using an elution buffer containing more than 40 mmoles per liter sodium cholate (19). Obviously, the detergent potency of endogenous bile salts is far less in native biles compared to model bile solutions in a given concentration range. The cause of this discrepancy is poorly understood but might be related to the presence of proteins in native bile. A decrease of the solubility of cholesterol by albumin has been observed in unconjugated bile salt solution (26). The mean vesicular cholesterol/phospholipid ratio of 2.0 in the 18 biles containing cholesterol crystals was not different from the mean vesicular cholesterol/phospholipid ratio in the three biles without cholesterol crystals. Similar ratios have been determined by Harvey et al. (4) in biles from patients with cholesterol gallstones as well as in biles from control patients without stones. It is unclear why some patients with prominent vesicular fractions in their biles do not develop cholesterol crystals, since it is not obviously related to the vesicle composition.

In conclusion, our study demonstrated a strong association between the presence of cholesterol-phospholipid vesicles and cholesterol monohydrate crystals in gall- 
bladder bile. The relative amount of vesicular cholesterol in gallbladder bile was not related to the cholesterol saturation index, the total lipid concentrations or the total bile salt concentration. Therefore, it was not possible to predict the physicochemical state of native bile sample (vesicle/micelle ratio) by measurements of the biliary lipid composition or the cholesterol saturation index. The strong association of vesicles and crystals in gallbladder bile is in accordance with the hypothesis that vesicles are important in the initiation of cholesterol crystallization. However, the importance of vesicles and crystals for the further development of cholesterol gallstones is still unclear, since the physicochemical properties of gallbladder bile obtained at cholecystectomy might not necessarily reflect the same properties of gallbladder bile at the time of gallstone formation.

Further studies in patients with recurrent gallstone disease after successful dissolution therapy may show whether the presence of vesicles or cholesterol crystals in bile could be an early reflection of the lithogenicity of bile.

Acknowledgments: The authors would like to thank Benedikta Zündt for her excellent technical assistance. We thank Dr. Ekkehard Pratschke for providing us with the samples.

\section{REFERENCES}

1. Mazer N, Carey MC. Quasi-elastic light-scattering studies of aqueous biliary lipid systems. Cholesterol solubilization and precipitation in model bile solutions. Biochemistry 1983; 22:426-442.

2. Sömjen GJ, Gilat T. A non-micellar mode of cholesterol transport in human bile. FEBS Lett 1983; 156:265-268.

3. Halpern Z, Dudley MA, Kibe A, et al. Rapid vesicle formation and aggregation in abnormal human biles. A time-lapse video-enhanced contrast microscopy study. Gastroenterology 1986; 90:875-885.

4. Harvey RPC, Sömjen G, Lichtenberg MS, et al. Nucleation of cholesterol from vesicles isolated from biles of patients with and without cholesterol gallstones. Biochim Biophys Acta 1987; 921:198-204.

5. Collins JJ, Phillips MC. The stability and structure of cholesterol rich codispersions of cholesterol and phosphatidylcholine. J Lipid Res 1982; 23:291-298.

6. Halpern Z, Dudley MA, Lynn MP, et al. Vesicle aggregation in model systems of supersaturated bile: relation to crystal nucleation and lipid composition of the vesicular phase. J Lipid Res 1986; 27:295-306.

7. Small DM. Cholesterol nucleation and growth in gallstone formation. N Engl J Med 1980; 302:1305-1307.

8. Sedaghat A, Grundy SM. Cholesterol crystals and the formation of cholesterol gallstones. N Engl J Med 1980; 302:1274-1277.

9. Poupon R, Chretin Y, Darnis F. Cristeaux de cholesterol, saturation de bile en cholesterol et lithiase biliaire. Gastroenterol Clin Biol 1984; 8:260-263.

10. Van der Linden W, Nakayama F. Occurrence of cholesterol crystals in human bile. Gut 1974; 15:630-635.

11. Holan KR, Holzbach RT, Hermann RE, et al. Nucleation time: a key factor in the pathogenesis of cholesterol gallstone disease. Gastroenterology 1979; 77:611-617.

12. Burnstein MJ, Ilson RG, Petrunka CN, et al. Evidence for a potent nucleating factor in the gallbladder bile of patients with cholesterol gallstones. Gastroenterology 1983; 85:801-807.

13. Harvey PRC, Sömjen G, Gilat T, et al. Vesicular cholesterol in bile. Relationship to protein concentration and nucleation time. Biochim Biophys Acta 1988; 958:10-18.

14. Tera $\mathrm{H}$. Stratification of human gallbladder bile in vivo. Acta Chir Scand 1960; 256(Suppl.):4-85.

15. Abell LL, Levy BB, Brodie BB, et al. A simplified method for the estimation of total cholesterol in serum and demonstration of its specificity. J Biol Chem 1952; 195:357-366.

16. Fiske $\mathrm{CH}$, Subbarow Y. The colorimetric determination of phosphorus. J Biol Chem 1925; 66:375-400.

17. Talalay P. Enzymatic analysis of steroid hormones. Biochem Anal 1960; 8:119-143.

18. Carey MC. Critical tables for calculating the cholesterol saturation of native bile. J Lipid Res 1978; 19:945-955.

19. Sömjen GJ, Gilat T. Contribution of vesicular and micellar carriers of cholesterol transport in human bile. J Lipid Res 1985; 26:699704.

20. Admirand WH, Small DM. The physico-chemical basis of cholesterol gallstone formation in man. J Clin Invest 1968; 47:1043-1052.

21. Carey MC, Small DM. The physical chemistry of cholesterol solubility in bile. J Clin Invest 1978; 61:998-1026.

22. Pattinson NR. Solubilisation of cholesterol in human bile. FEBS Lett 1985; 181:339-342.

23. Pattinson NR, Chapman BA. Distribution of biliary cholesterol between mixed micelles and nonmicelles in relation to fasting and feeding in humans. Gastroenterology 1986; 91:697-702.

24. Ulloa N, Garrido J, Nervi F. Ultracentrifugal isolation of vesicular carriers of biliary cholesterol in native human and rat bile. Hepatology 1987; 7:235-244.

25. Whiting MJ, Watts JM. Supersaturated bile from obese patients without gallstones supports cholesterol crystal growth but not nucleation. Gastroenterology 1984; 86:243-248.

26. Fukudome K, Chijiiwa K, Furusawa T, et al. Effect of albumin on the solubility of cholesterol in bile. Biochim Biophys Acta 1987; 922:155-161. 